\title{
Instituição de um Protocolo de Vigilância Alimentar e Nutricional no Município de Jaguari - RS
}

\author{
Hailê Zeul Panisson Taschetto, ${ }^{1}$ Teles Medeiros, ${ }^{2}$ Regina Gema Santini Costenaro, ${ }^{1}$ \\ Léris Salete Haeffner, ${ }^{1}$ Franceliane Jobim Benedetti ${ }^{3}$
}

\begin{abstract}
RESUMO
Os controles periódicos antropométricos do Programa Bolsa Família devem seguir os passos preconizados pelo protocolo do Sistema de Vigilância Alimentar e Nutricional. Observou-se, porém, que este protocolo não estava sendo aplicado em sua plenitude no município de Jaguari-RS, perdendo-se a oportunidade de identificar as crianças com distúrbios nutricionais e tomar as medidas pertinentes a cada caso. Com o objetivo de instituir um protocolo de Vigilância Alimentar e Nutricional que seja efetivamente aplicado, foi desenvolvida uma pesquisa-ação. Após, feito o diagnóstico do funcionamento/operacionalidade do Sistema de Vigilância Alimentar e Nutricional e conhecida a situação nutricional das crianças beneficiárias do programa, público-alvo, foram feitas alterações operacionais nas avaliações antropométricas semestrais e realizada capacitação antropométrica dos profissionais de saúde. A monitorização inicial, realizada durante um período de controle antropométrico do Programa Bolsa Família, revelou resultados positivos no estabelecimento do protocolo de Vigilância Alimentar e Nutricional. Palavras-chave: Criança. Política pública. Avaliação nutricional.

IMPLEMENTATION OF A FOOD AND NUTRITIONAL SURVEILLANCE PROTOCOL IN THE MUNICIPALITY OF JAGUARI - RS ABSTRACT

Regular nutritional assessment is one condicionality of the Bolsa Familia Program that must follow all recommended steps of Brazilian Food and Nutrition Surveillance System protocol. However, it was observed that protocol was not accomplished in all extension in the city of Jaguari- RS, south of Brazil. That way, it was leaving out the opportunity for identifying children nutritional disorders and act in order to take adequate measures. An action research was performed in order to effectively implement a food and nutrition surveillance protocol. First, it was verified the way Food and Nutrition Surveillance System was been operated and it was also checked nutritional status from children enrolled in program. Then, operating changings were set up in anthropometric assessments and it was realized a training in anthropometry for health professionals. Monitoring performed during an anthropometric assessment showed positive results in the process of food and nutrition surveillance protocol implementation.
\end{abstract}

Keywords: Child. Public policy. Nutrition assessment.

\footnotetext{
Universidade Franciscana. Santa Maria/RS, Brasil.

2 Prefeitura de Jaguari. Jaguari/RS, Brasil.

${ }^{3}$ Autora correspondente. Universidade Franciscana - UFN. Rua Silva Jardim, 1175, Nossa Senhora do Rosário, Santa Maria/RS, Brasil. CEP 97010-491. http:// lattes.cnpq.br/5711764386647641.https://orcid.org/0000-0002-3334-3910 francijb@yahoo.com.br
} 


\section{INTRODUÇÃO}

Os dados coletados por ocasião do monitoramento de peso e estatura das crianças beneficiárias do Programa Bolsa Família (PBF), devem ser repassados para o Sistema de Vigilância Alimentar e Nutricional (Sisvan) (BRASIL, 2008). Embora, em muitos municípios, apenas os dados do PBF, por serem de registro obrigatório, sejam fornecidos ao sistema (DAMÉ et al., 2011), os protocolos do Sisvan recomendam a Vigilância Alimentar e Nutricional (VAN) para todos os grupos populacionais. As informações, provenientes dos municípios que alimentam o sistema, são integradas em âmbito nacional e são gerados mapas e relatórios informando o estado nutricional da população brasileira. Estes dados servem de subsídios para o estabelecimento de políticas públicas que possibilitem a prevenção e a reversão dos distúrbios nutricionais. Além dos registros dos dados, os protocolos do Sisvan na assistência à saúde recomendam também que o profissional, que esteja coletando os dados da criança, faça as anotações dos dados nos gráficos da Caderneta de Saúde da Criança (CSC), firme um diagnóstico nutricional, comunique-o aos responsáveis da criança e faça as intervenções adequadas a cada caso (BRASIL, 2008).

No processo de estabelecimento de um protocolo, é fundamental que se conheçam as peculiaridades do público-alvo das ações de saúde e deve haver consenso e equilíbrio entre o que é embasado pelas evidências científicas e o que é necessário e possível em cada unidade de saúde. É importante, também, levar em consideração que as ações em saúde, desenvolvidas nos serviços, acontecem tanto com base no conhecimento científico e tecnológico quanto nas vivências e valores dos profissionais que as planejam e executam. A qualidade da ação desenvolvida vai depender do conhecimento, do compromisso, da implicação e do valor atribuído ao serviço pelo trabalhador (WERNECK; FARIA; CAMPOS, 2009).

Observando-se as avaliações periódicas das crianças do PBF no município de Jaguari, integrante da 4a Coordenadoria Regional de Saúde do Estado do Rio Grande do Sul (4a CRS-RS), verificaram-se problemas operacionais importantes e o principal deles era a não execução do protocolo do Sisvan na sua integralidade. Assim perdia-se a oportunidade de fazer o diagnóstico nutricional das crianças e de intervir se necessário. Com a intenção de mudar este cenário, este trabalho tem por objetivo introduzir um protocolo de VAN para as crianças beneficiárias do PBF.

\section{MÉTODO}

O presente trabalho foi realizado no município de Jaguari, que pertence à região de saúde Entre Rios da 4a CRS-RS. O município está localizado na região centro-oeste do Estado do Rio Grande do Sul. A população atual é de cerca de 11.000 habitantes (IBGE, 2010). A população-alvo direta foi os profissionais de saúde que trabalham nas três unidades de saúde (US) do município. Como população indireta, crianças menores de cinco anos cadastradas no PBF de Jaguari.

Esse trabalho fundamentou-se numa pesquisa-ação, a qual foi constituída das seguintes fases:

- 1a fase: identificação do problema: o não uso do protocolo do Sisvan nas avaliações periódicas das crianças do PBF.

- 2a fase: planejamento para a efetivação de um protocolo de VAN para as avaliações do PBF. Para executar esta ação foram levadas em consideração três condições básicas: o uso do protocolo do Sisvan como referencial; participação ativa dos profissionais de saúde das Unidades de Saúde (US) neste processo; e conhecimento do estado nutricional antropométrico das crianças beneficiárias do PBF, público-alvo do protocolo.

Seguiu-se, assim, quatro passos, descritos a seguir:

- Passo 1: avaliar como o protocolo do Sisvan estava sendo aplicado nos controles antropométricos semestrais do PBF, qual o conhecimento das técnicas antropométricas pelos profissionais de saúde e como o protocolo do Sisvan era aplicado nas outras ações em saúde nas US. Para cumprir com estes objetivos foram utilizadas as observações realizadas durante o período de exercício profissional na US, e foi realizada uma pesquisa descritiva, construída com dados primários obtidos de questionário aplicado aos profissionais das US do município de Jaguari. O questionário continha 12 perguntas abordando os seguintes tópicos: participação em capacitações sobre antropometria; ambiente de realização da coleta dos dados antropométricos; técnica antropométrica; preenchimento da CSC; realização de diagnóstico nutricional e informação deste aos familiares; encaminhamento das crianças com distúrbios nutricionais; e aferição dos equipamentos usados pelo Inmetro (SILVA, 2013). A coleta de dados foi realizada no mês de dezembro de 2017.

- Passo 2: em março de 2018 foi realizada uma explanação sobre o Sisvan, com o objetivo de capacitar os profissionais de saúde para as discussões sobre o protocolo. Inicialmente, para verificar o grau de conhecimento sobre o assunto, foi realizada uma 
pré-avaliação escrita. Depois, por meio de exposição com Datashow, foram abordados especialmente os dez passos do Sisvan, que orientam para a coleta e análise de dados antropométricos em serviços de saúde. Foram também apresentados gráficos de peso e estatura e discutido com os participantes qual era a situação nutricional da criança. No final, foi aplicada uma pós-avaliação escrita, para verificar o conhecimento adquirido.

- Passo 3: conhecer a evolução do estado nutricional antropométrico das crianças beneficiárias do PBF do município de Jaguari, por ser o local da instalação do protocolo de VAN. Com este objetivo foi realizada uma pesquisa descritiva longitudinal construída com dados de relatórios públicos consolidados do Sisvan WEB (BRASIL, 2017). A população do estudo foi constituída de crianças menores de cinco anos de idade, de famílias beneficiárias do PBF. Foram levados em consideração os índices antropométricos peso/idade $(P / I)$, estatura/idade (E/I) e IMC/idade (IMC/I), colhidos no período de 2008 a 2017. Além dos dados de Jaguari, foram também colhidos dados do Sisvan da 4a CRS-RS para comparação.

- Passo 4: em abril de 2018, realizou-se uma roda de conversa com os profissionais de saúde para discutir a viabilidade do protocolo de VAN.

- 3a fase: ações para o estabelecimento de um protocolo de VAN: definição de mudanças na operacionalização das avaliações semestrais antropométricas do PBF e capacitação dos profissionais. Destaca-se que, neste estudo, o protocolo restringiu-se à avaliação antropométrica; o perfil e avaliação do consumo alimentar não foram contemplados.

- 4a fase: monitorização e descrição dos efeitos da instalação do protocolo de VAN: os dados foram anotados em um diário de campo sob a forma de texto com as observações da aplicabilidade do protocolo. Os tópicos norteadores foram: o grau de aderência dos profissionais; o tempo de espera do usuário para atendimento; o uso de local adequado; o uso apropriado da técnica antropométrica; registro dos dados na CSC com anotação nos gráficos; realização do diagnóstico nutricional e informação deste para a mãe; e encaminhamento das crianças em risco nutricional.

\section{Análises estatísticas}

Os dados foram digitados no programa Microsoft Excel e organizados em tabelas e gráfico. Para a análise dos dados foi utilizado o programa Statistical Package for the Social Sciences (SPSS) versão 23.0.
Mediante apresentação do Projeto de Pesquisa, o mesmo foi analisado e autorizado pela secretária municipal de saúde do município de Jaguari. Obteve-se, também, parecer favorável da Comissão de Ética em Pesquisa, em conformidade com a Resolução 466/2012 do CNS/MS, inscrito sob o Certificado de Apresentação para Apreciação Ética (CAAE) de número 80290017.4.0000.5306. Os Termos de Consentimento Livre e Esclarecido (TCLE) foram assinados pelos profissionais de saúde que participaram da pesquisa, caracterizando a voluntariedade da participação.

\section{RESULTADOS E DISCUSSÕES}

Os resultados e discussões serão apresentados em tópicos conforme as fases e passos expostos na metodologia.

\section{Diagnóstico do funcionamento/operacionalidade} do protocolo do Sisvan

A observação dos controles antropométricos periódicos do PBF evidenciou que eles eram realizados numa única US, em dias e horários predeterminados, num período máximo de duas semanas. Um profissional da área administrativa, responsável pelo PBF, era quem coletava os dados e os repassava ao Sisvan. No período inicial, de maior demanda, um outro profissional (nem sempre o mesmo) da área administrativa, também participava da coleta dos dados antropométricos. Eventualmente, participava algum profissional da área da enfermagem. As crianças eram pesadas e medidas sem serem despidas, numa sala improvisada e sem privacidade. No salão de espera, localizado na parte da frente do prédio, e mesmo na área externa da US, havia a formação de filas nos dias de controle antropométrico das crianças beneficiárias do PBF. O procedimento encerrava na anotação dos dados coletados na ficha do Sisvan, com posterior digitação pela funcionária responsável no Sisvan WEB. Assim, verificou-se que não eram executados os demais passos preconizados pelo protocolo do Sisvan, ou seja, não havia anotação dos dados antropométricos na CSC, deixava-se de realizar o diagnóstico nutricional e perdia-se a oportunidade de realizar alguma intervenção nas crianças com distúrbios nutricionais.

A análise dos dados dos questionários aplicados aos sete profissionais de saúde das USs do município de Jaguari, que concordaram em participar da pesquisa, evidenciou as informações descritas na Tabela 1 e complementadas no texto. 
Tabela 1 - Descrição do conhecimento dos profissionais de saúde sobre as técnicas antropométricas do protocolo Sisvan

\begin{tabular}{lccc}
\hline \multicolumn{1}{c}{ Variáveis } & Sim & Não & $\begin{array}{c}\text { Às vezes/ } \\
\text { Não sabe }\end{array}$ \\
\hline Participação em capacitação & $57,1 \%(4)$ & $42,9 \%(3)$ & \\
Ambiente adequado & $71,5 \%(5)$ & $28,5 \%(2)$ & \\
Técnica & & & \\
Pesagem com roupa & $28,5 \%(2)$ & $71,5 \%(5)$ & \\
Uso balança pediátrica & $57,1 \%(4)$ & $42,9 \%(3)$ & \\
Posicionamento adequado & $57,1 \%(4)$ & $42,9 \%(3)$ & \\
Anotação na CSC & $57,1 \%(4)$ & $42,9 \%(3)$ & $0 \%$ \\
Diagnóstico Nutricional & $42,9 \%(3)$ & $28,5 \%(2)$ & $28,5 \%(2)$ \\
Aferição dos equipamentos & $85,7 \%(6)$ & $0 \%$ & $14,36 \%(1)$ \\
\hline
\end{tabular}

CSC; Caderneta de Saúde da Criança. Valores apresentados em \% (n).

Fonte: Elaborado pelos autores.

\section{Capacitações sobre antropometria}

$57,1 \%$ dos entrevistados $(n=4)$ informa ter participado de alguma capacitação sobre como medir e pesar adequadamente as crianças. Dos entrevistados que já realizaram capacitação antropométrica, a metade $(n=2)$ revela tê-la realizada há três anos ou mais (um respondeu que realizou há mais de três anos e outro não respondeu à questão). A participação periódica e regular do profissional de saúde em capacitações e treinamentos em antropometria melhora a qualidade de suas medidas, e possibilita um diagnóstico nutricional mais próximo do real. Muitos profissionais estudaram as técnicas antropométricas apenas em seu curso de formação e, como raramente participam de treinamentos ou reciclagens na área, estão sujeitos a coletar medidas pouco fidedignas. Isso pode levar à formulação de falsos diagnósticos nutricionais, tanto para mais quanto para menos (ULIJASZEK; KERR, 1999; BAGNI; BARROS, 2015).

\section{Ambiente de coleta dos dados antropométricos}

Dos entrevistados, $71,5 \%(n=5)$ responderam que a coleta é realizada numa sala específica para esta finalidade, com aquecimento no inverno; $28,5 \%(n=2)$ responderam que o local da coleta dos dados antropométricos é uma sala ou salão improvisado, sem privacidade, normalmente usada para outras atividades. Esta última situação ocorre durante as avaliações das crianças do PBF. Em pesquisa realizada em quatro municípios pertencentes ao Departamento Regional de Saúde VI de Bauru-SP, observou-se que $67 \%$ dos municípios dispunham de sala própria para a realização de antropometria (FERREIRA et al., 2017).

\section{Técnica antropométrica}

Dos entrevistados, $28,5 \%$ ( $n=2)$ não despem as crianças para pesá-las. Um percentual de $42,9 \%(n=3)$ afirma que a criança pode ser pesada no colo da mãe e que também pode ser medida de pé, mesmo tendo menos de dois anos de idade, desde que tenha habilidade para ficar nesta posição. Os profissionais que realizam a coleta dos dados antropométricos costumam cometer erros frequentes que comprometem a confiabilidade dos resultados (FERREIRA et al., 2017; SILVA et al., 2017). Um estudo encontrou uma diferença significativa ( $p: 0,0015$ ) média de $26,5 \mathrm{~g}$ entre o peso direto na balança pediátrica e o indireto no colo da mãe, mesmo as crianças sendo pesadas pelo mesmo profissional e fazendo-se a calibração prévia das balanças (CAINO et al., 2006).

\section{Preenchimento da CSC}

Apesar de a maioria responder que anota sempre os dados coletados nos gráficos de peso e estatura da CSC, uma parcela expressiva $(42,9 \%, n=3)$ nem sempre o faz. Mesmo assim, os resultados são bem melhores do que os encontrados na literatura. Palombo et al. (2014) encontraram um percentual muito baixo $(8,9 \%)$ de preenchimento dos gráficos de peso e estatura, corroborando resultados de estudos semeIhantes (ABUD; GAIIVA, 2015; PEDRAZA, 2019; MOREIRA; GAÍVA, 2013). Observa-se, então, que os profissionais de saúde valorizam pouco o uso da CSC como instrumento de acompanhamento da saúde, nutrição e desenvolvimento infantil (ABREU; VIANA; CUNHA, 2012). Questionados sobre o motivo de não preenchimento adequado da CSC, todos os entrevistados desta pesquisa afirmam que isso ocorre porque a mãe não leva a caderneta às USs. O não preenchimento ou o preenchimento incompleto da CSC fazem com que as mães pensem que a caderneta seja pouco importante no acompanhamento da criança; assim, não são levadas às unidades de saúde e são mal conservadas (PALOMBO et al., 2014). Outras razões apontadas na literatura para a utilização incorreta e o preenchimento inadequado da CSC, como a falta de divulgação, de treinamento ou de algum tipo de capacitação e o despreparo dos profissionais de saúde para lidar com os novos conceitos incorporados à caderneta, como as curvas de referência em escore-z e o gráfico de IMC/ idade (ABREU; VIANA; CUNHA, 2012; FREITAS et al., 2019), não foram referidos pelos entrevistados desta pesquisa. 


\section{Realização do diagnóstico nutricional e informação aos pais}

Dos entrevistados, $42,9 \%(n=3)$ responderam que sempre realizam o diagnóstico nutricional; $28,5 \%$ $(n=2)$ afirmam que nem sempre, e igual porcentagem revela que nunca realiza o diagnóstico nutricional. Quando este é feito, $60 \%(n=3)$ repassam sempre as informações do diagnóstico nutricional das crianças aos familiares, mas os restantes $(40 \%, n=2)$ nem sempre o fazem.

\section{Encaminhamento das crianças com distúrbios nutricionais}

Dos entrevistados que realizam o diagnóstico nutricional, $57,14 \%(n=4)$ responderam que encaminham as crianças com distúrbios nutricionais para consulta médica. Apenas um profissional referiu que encaminha para o médico e nutricionista.

\section{Aferição dos equipamentos}

Dos entrevistados, $85,7 \%(n=6)$ responderam que os equipamentos são aferidos pelo Inmetro. Em estudo realizado em Bauru-SP, os pesquisadores observaram que as balanças não apresentavam condições adequadas de calibração em $29 \%$ e $20 \%$ das unidades de metade dos municípios que participaram do estudo (FERREIRA et al., 2017).

\section{Pesquisa sobre o estado nutricional das crianças} beneficiárias do PBF de Jaguari

Foram coletados dados do Sisvan WEB de crianças menores de cinco anos beneficiárias do PBF do município de Jaguari, no período de 2008 a 2017. Os valores referentes aos indicadores $\mathrm{P} / \mathrm{I}, \mathrm{IMC} / \mathrm{I}$ e $\mathrm{E} / \mathrm{I}$ estão apresentados nos gráficos 1 , 2, e 3 . Observa-se diminuição nos estados de magreza e baixa estatura, porém aumento do excesso de peso entre 2008 e 2017.

Gráfico 1 - Estado nutricional de crianças de zero a 5 anos beneficiárias do PBF de Jaguari, de acordo com o Índice Peso para Idade, no período de 2008 a 2017

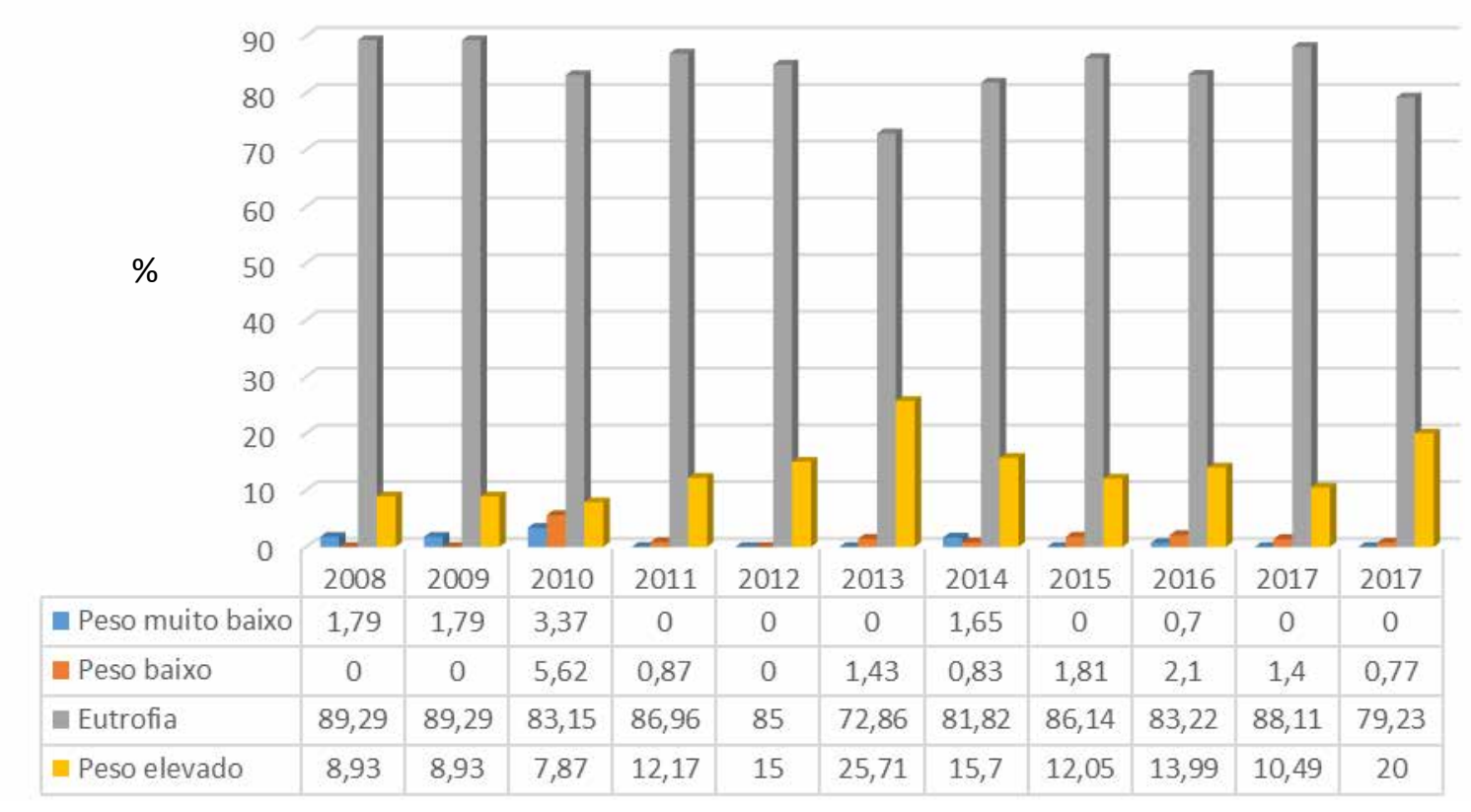


Gráfico 2 - Estado nutricional de crianças de zero a 5 anos beneficiárias do PBF de Jaguari, de acordo com o Índice de Massa Corporal (IMC) para Idade, no período de 2008 a 2017

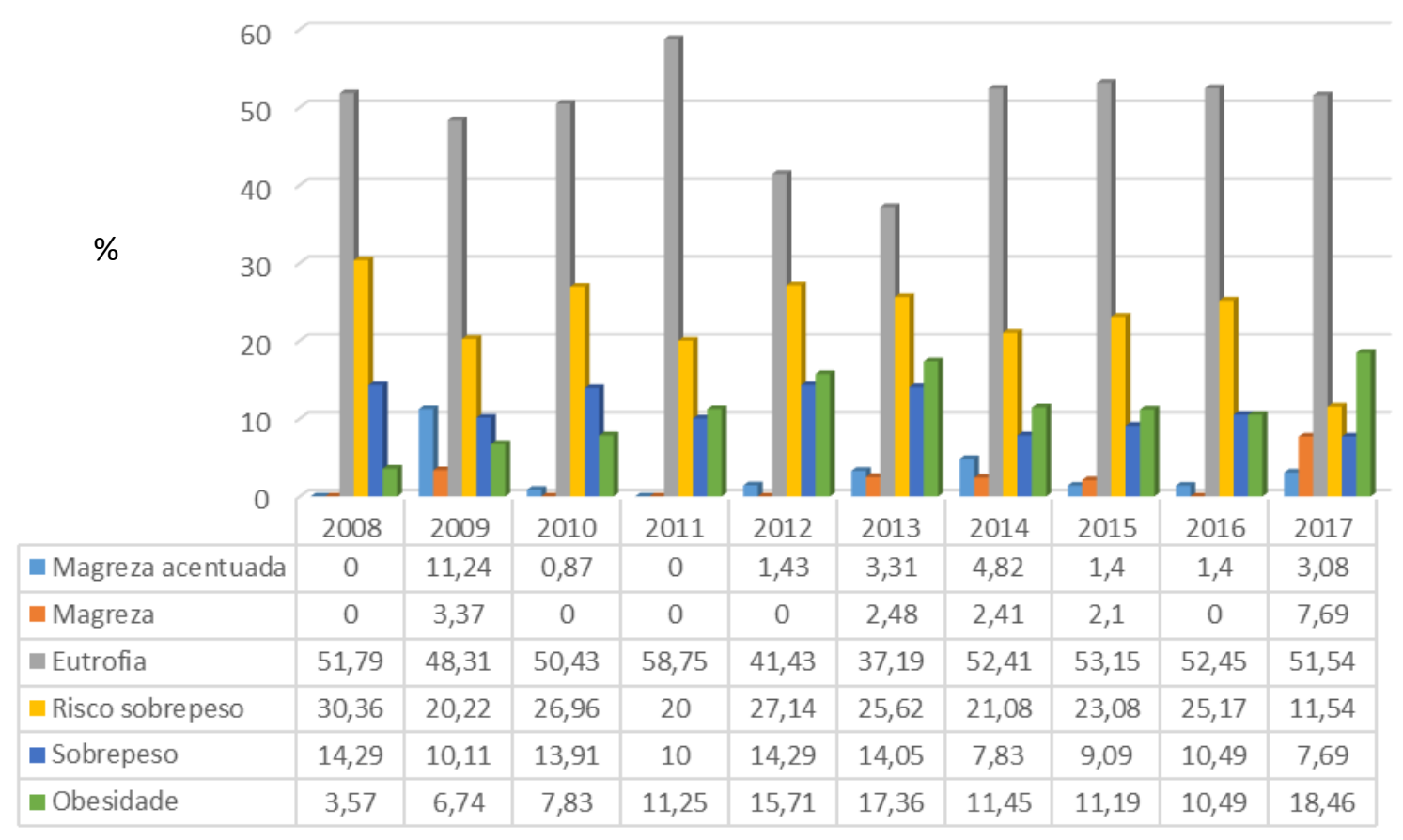

Valores apresentados em percentuais.

Fonte: Elaborado pelos autores.

Gráfico 3 - Altura de crianças de zero a 5 anos beneficiárias do PBF de Jaguari, de acordo com o Índice Estatura para Idade, no período de 2008 a 2017

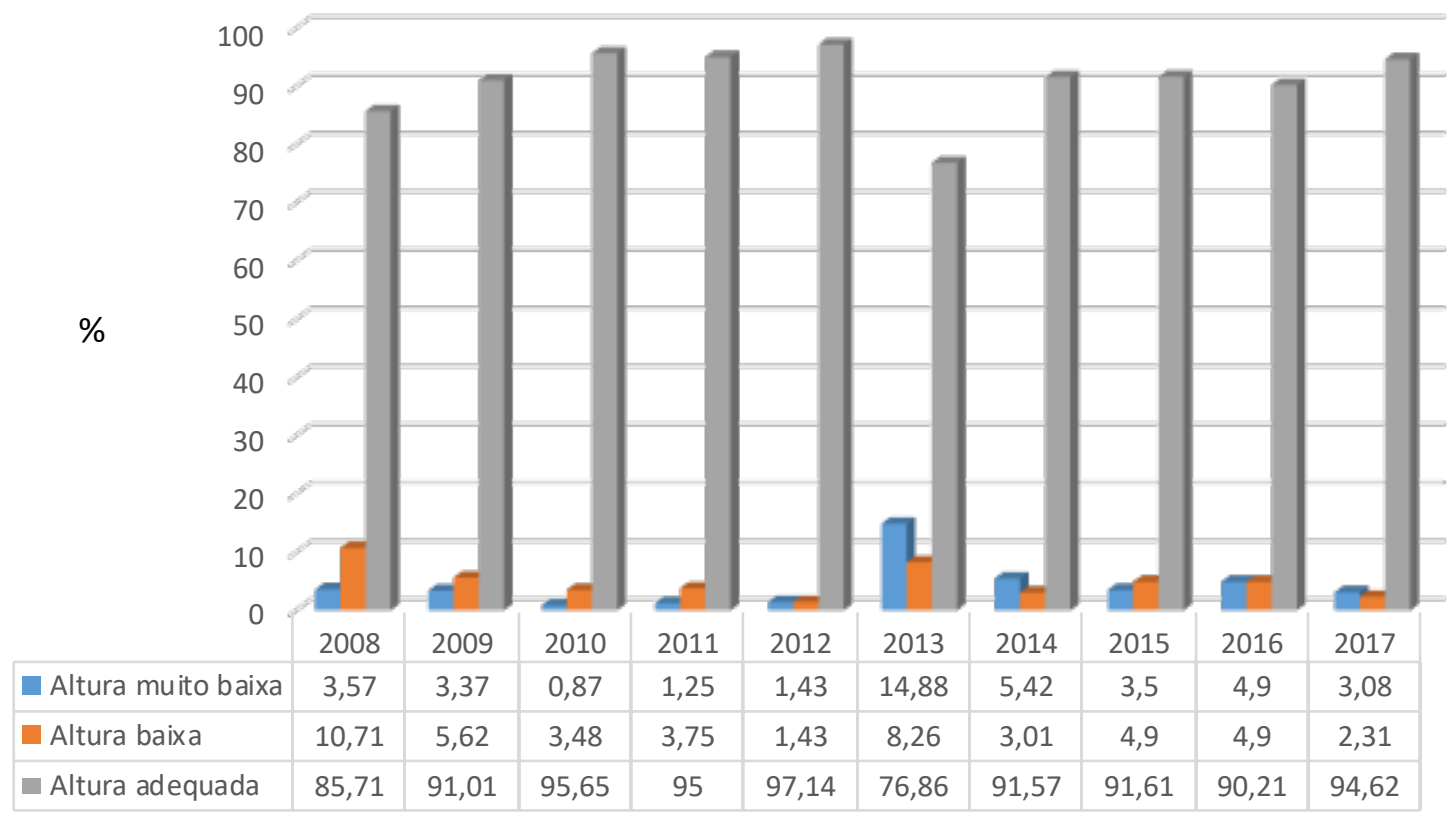

Valores apresentados em percentuais.

Fonte: Elaborado pelos autores. 


\section{Capacitação sobre o protocolo do Sisvan}

Além dos técnicos de enfermagem e enfermeiras das US, participaram da capacitação também agentes de saúde, perfazendo um total de 16 profissionais. Na avaliação do desempenho da pré-avaliação, apenas três participantes da capacitação obtiveram nota 6 ou mais. A mediana das notas foi 4 (2-4).

O mau desempenho na pré-avaliação confirma o desconhecimento das normas técnicas e a falta de preparo dos profissionais de saúde para a coleta apropriada das medidas antropométricas para a marcação nos gráficos das CSC e a correta realização do diagnóstico nutricional das crianças avaliadas. Dentre os erros mais frequentes relacionados à coleta dos dados antropométricos, destacam-se: a não calibração da balança antes de iniciar a pesagem dos menores de 24 meses; a pesagem de crianças menores de dois anos em balança antropométrica de plataforma mecânica; as crianças não são despidas corretamente antes da aferição do peso; não aguardar o alinhamento da aguIha do braço e o fiel da balança antes da leitura; leitura do resultado obtido em balança pediátrica mecânica realizada de forma incorreta; e posicionamento inadequado do corpo das crianças de 24 a 60 meses para a aferição da altura (FERREIRA et al., 2017; SILVA et al., 2017). O mau desempenho dos profissionais de saúde nas questões relativas a marcação dos dados antropométricos nos gráficos das CSC e na interpretação dos mesmos, pressupõe-se que seja porque essa atividade não faça parte da sua rotina nas US. Os profissionais de saúde não valorizam muito o uso da CSC como instrumento de acompanhamento da saúde, nutrição e desenvolvimento infantil (ABREU; VIANA; CUNHA, 2012, GONÇALVES et al., 2020). Palombo et al. (2014) encontraram um percentual muito baixo $(8,9 \%)$ de preenchimento dos gráficos de peso e estatura, corroborando resultados de estudos semelhantes (PEDRAZA, 2019; MOREIRA; GAÍVA, 2013). O despreparo dos profissionais de saúde para lidar com os novos conceitos incorporados à caderneta, como as curvas de referência em escore-z e o gráfico de IMC-para-idade, poderia justificar, em parte, o baixo percentual de preenchimento dos gráficos de crescimento (ABREU; VIANA; CUNHA, 2012).

Na avaliação do desempenho da pós-avaliação, apenas um dos participantes obteve nota abaixo de 6 . A média das notas foi 8,81 ( $\pm 1,56 \mathrm{DP})$, demonstrando que a capacitação realizada conseguiu seu objetivo de melhorar o nível de conhecimento dos participantes sobre coleta de dados antropométricos e o uso correto das CSC no acompanhamento da saúde, crescimento e desenvolvimento das crianças.

\section{Ações para a introdução de um protocolo de VAN}

As ações foram de dois tipos, a seguir descritas:

- Mudanças operacionais: em março de 2018 foram postas em ação as seguintes medidas: as avaliações só serão realizadas pelos profissionais de enfermagem; o período de avaliações foi estendido para dois meses, sem datas preestabelecidas; as avaliações serão realizadas nas três USs de Jaguari, conforme sua área de abrangência; uso de salas adequadas, com controle ambiental de temperatura, permitindo condições apropriadas de conforto térmico e privacidade para que as crianças possam ser despidas para a correta aferição das medidas antropométricas; e afixação nas salas de pesagem e medição de banners com o fluxograma de VAN e de tabelas de classificação nutricional.

- Capacitação dos profissionais de saúde: em março de 2018 foi realizada uma capacitação com todos os profissionais de saúde das unidades de saúde de Jaguari sobre o protocolo de VAN. Na ocasião foram apresentados os resultados da pesquisa sobre o estado nutricional das crianças beneficiárias do PBF de Jaguari, as mudanças operacionais nas avaliações do PBF e o fluxograma (Figura 1) a ser seguido nas avaliações antropométricas.

\section{Monitorização e descrição dos efeitos da institui-} ção do protocolo de VAN

A monitorização foi realizada no primeiro semestre de 2018. Observações pontuais realizadas nas três US evidenciaram resultados positivos nas avaliações do PBF. As crianças beneficiárias do PBF foram incluídas no fluxo normal de atendimento, não havendo formação de filas. As avaliações foram realizadas pelos profissionais de enfermagem. As salas usadas para as avaliações do PBF foram as mesmas utilizadas para a triagem das consultas pela enfermagem, com condições adequadas de conforto e privacidade. De um modo geral, percebeu-se uso de técnica adequada de coleta dos dados antropométricos, os quais foram registrados nas CSCs, com a realização do diagnóstico nutricional e o devido repasse das informações aos responsáveis. $O$ sistema de encaminhamento das crianças com distúrbios nutricionais não se mostrou ainda devidamente estruturado, havendo apenas a orientação aos pais para que levassem as crianças ao médico. 
Figura 1 - Fluxograma de avaliação nutricional de crianças beneficiárias do PBF

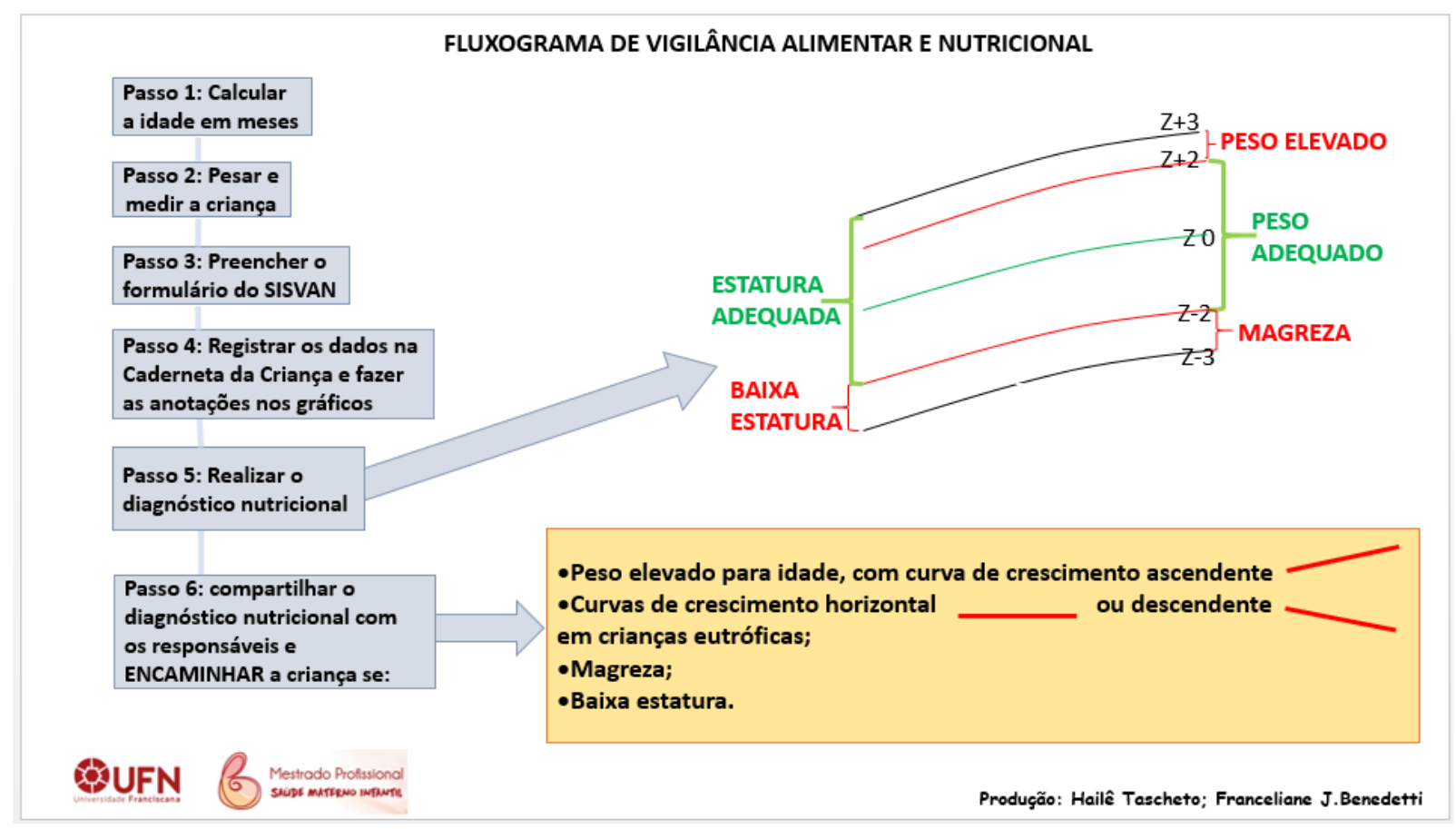

Fonte: Hailê Zeul Panisson Taschetto e Franceliane Jobim Benedetti.

\section{CONSIDERAÇÕES FINAIS}

A introdução do protocolo de VAN nas USs de Jaguari ocorreu de forma efetiva porque todos os fatores foram considerados atentamente neste processo: o conhecimento científico-tecnológico, a realidade local do serviço de saúde, o público beneficiado (crianças beneficiárias do PBF) e os agentes executores do protocolo (os profissionais de saúde). A partir da sensibilização dos profissionais de saúde para a necessidade do uso adequado de um protocolo de VAN, da identificação dos problemas operacionais no uso do protocolo do Sisvan e do conhecimento da situação nutricional das crianças beneficiárias do PBF, foram desencadeadas as ações para a execução do protocolo. Dentre estas ações, destacaram-se as capacitações dos profissionais de saúde, inicialmente para os processos de discussão e de elaboração e, finalmente, para o uso do protocolo de VAN.

Recomenda-se que sejam realizadas periodicamente capacitações dos profissionais de saúde e que estas ocasiões possam servir também para o compartilhamento de experiências, problemas e sugestões. Isso é importante para manter os profissionais motivados e engajados na continuidade da aplicação do protocolo de VAN, não só para os beneficiários do PBF, mas também para todas as situações em que ele é recomendado.

\section{REFERÊNCIAS}

ABREU, T. G. T.; VIANA, L. S.; CUNHA, C. L. F. C. Desafios na utilização da caderneta de saúde da criança: entre o real e o ideal, J Manag. Prim. Health Care, v. 3, n. 2, p. 80-83, 2012.

ABUD, S. M.; GAÍVA, M. A. M. Registro dos dados de crescimento e desenvolvimento na caderneta de saúde da criança. Rev. Gaúcha Enferm., v. 36, n. 2, p. 97-105, 2015.

BAGNI, U. V.; BARROS, D. C. de. Erro em antropometria aplicada à avaliação nutricional nos serviços de saúde: causas, consequências e métodos de mensuração. Nutrire, v. 40, n. 2, p. 226-236, ago. 2015.

BRASIL. Ministério da Saúde. Protocolos do Sistema de Vigilância Alimentar e Nutricional - Sisvan - na assistência à saúde. Brasília: Ministério da Saúde, 2008.

BRASIL. Ministério da Saúde. Vigilância Alimentar e Nutricional - Sisvan. Relatórios públicos do Sisvan. Brasília, DF: Ministério da Saúde, 2017. Disponível em: http://dabsistemas.saude.gov.br/sistemas/sisvanV2/relatoriopublico/ index.

CAINO, D. S. et al. Comparación de dos técnicas de medición de peso en niños menores de cuatro años. Arch. Argent. Pediatr, v. 104, n. 3, p. 217-220, 2006.

DAMÉ, P. K. V. et al. Sistema de Vigilância Alimentar e Nutricional (Sisvan) em crianças do Rio Grande do Sul, Brasil: cobertura, estado nutricional e confiabilidade dos dados. Cad. Saúde Pública, v. 27, n. 11, p. 2.155-2.165, 2011. 
FERREIRA, M. C. S. et al. Monitoramento nutricional em unidades de atenção primária à saúde. Rasbran - Revista da Associação Brasileira de Nutrição. São Paulo, ano 8, n. 1, p. 37-45, jan./jun. 2017.

FREITAS, J. L. G. et al. Preenchimento da caderneta de saúde da criança na primeira infância. Rev. Bras. Promoç. Saúde, v. 32, p. 8.407, 2019.

GONÇALVES, I. S. A. et al. Nutritional status coverage trend registered in the SISVAN web in seven municipalities of the Zona da Mata Mineira, Brazil, from 2008 to 2017, and its association with socio-economic, demographic and organisation of health system variables. Journal of Nutritional Science, v. 9, e4, p. 1-8, 2020.

IGBE. Instituto Brasileiro de Geografia e Estatística. Censo Demográfico 2010. 2010. Disponível em: https://sidra.ibge. gov.br/pesquisa/censo-demografico/demografico-2010/inicial. Acesso em: abr. 2020.

MOREIRA, M. D. S.; GAÍVA, M. A. M. Acompanhamento do crescimento e desenvolvimento infantil: análise dos registros das consultas de enfermagem. Rev. Pesqui. Cuid. Fundam., v. 5, n. 2, p. 3.757-3.766, 2013.

PALOMBO, C. N. T. et al. Uso e preenchimento da caderneta de saúde da criança com foco no crescimento e desenvolvimento. Rev. Esc. Enferm., USP, v. 48, p. 59-66, 2014.

PEDRAZA, F. D. Preenchimento da caderneta de saúde da criança e antropometria de crianças. J Manag. Prim. Health Care, v. 10, e10, 2019.

SILVA, G. A. S. et al. Procedimentos de medição da massa corporal infantil pelos agentes comunitários de saúde de Macaé, Rio de Janeiro, 2010-2011. Epidemiol. Serv. Saúde, v. 26, n. 3, jul./set. 2017.

SILVA, S. T. Vigilância alimentar e nutricional: proposta de protocolo. Monografia (Especialização em Residência Multiprofissional em Atenção Básica/Saúde da Família) - Universidade do Extremo Sul Catarinense - Unesc, Criciúma, 2013.

ULIJASZEK, S. J.; KERR, D. A. Review article Anthropometric measurement error and the assessment of nutritional status. British Journal of Nutrition, v. 44, p. 165-177, 1999.

VITOLO, M. R.; GAMA, C. M.; CAMPAGNOLO, P. D. B. Frequência de utilização do serviço público de puericultura e fatores associados. J. Pediatr, v. 86, n. 1, p. 80-84, 2010.

WERNECK, M. A. F.; FARIA, H. P.; CAMPOS, K. F. C. Protocolo de cuidados à saúde e de organização do serviço. Belo Horizonte: Nescon; UFMG; Coopmed, 2009. 